\title{
PROBLEMES DE CONTROLE OPTIMAL EN PHYSIQUE DES PLASMAS
}

Détermination d'un équilibre dans un Tokomak

\author{
par \\ J.P. BOUJOT, J.P. MORERA, R. TEMAM ${ }^{*}$ \\ 17 - 21 décembre 1973 \\ IRIA
}

Compagnie Internationale de Services en Informatique (C.I.S.I.)

Commissariat à l'Energie Atomique - SACLAY

*

Aussi Institut de Recherche en Informatique et Automatique (I.R.I.A.) 


\section{INTRODUCTION}

Le Tokomak est une des machines les plus prometteuses actuellement envisagée pour rêaliser la fusion contrôlée. Il se campose schématiquement d'une coque toroïdale contenant un plasma que I'on cherche à chauffer intensément sans introduire d'instabilités. Il semble que la forme de la section droite du plasma ait précisément une très grande importance en ce qui concerne la stabilité de l'anneau de plasma et son chauffage. Il semble acquis qu'une forme elliptique est plus avantageuse qu'une section circulaire.

Un problème important se trouve donc être l'étude de dispositifs permettant de donner au plasma une configuration préalablement choisie.

Claude Mercier et Soubbaramayer du Commissariat à l'Energie Atomique ${ }^{(1)}$ proposent pour celâ d'introduire dans la cavité entre le plasma ef la coque ${ }^{(2)}$, des conducteurs électriques: il faut choisir les courants de decharge tel que le plasma soit en équilibre dans la configuration souhaitée. Notre objet est d'exprimer ce problème, comme un problème de commande optimale que nous formulerons et étudierons dans la suite. L'étude que nous présentons a été menée en étroite collaboration avec le S.T.G.I., en particulier Claude Mercier et Soubbaramayer qui ont jové un rôle déterminant dans la formulation du problème.

Dans le premier paragraphe nous décrivons brièvement :

Le Tokomak

. Les équations régissant le dispositif (on a choisi un modèle linéaire stationnaire)

. Le problème physique lui-même.

Les deuxième et troisième paragraphes contiennent la formulation et l'étude de différents problèmes de contrôle optimal. La dernière partie contient une description succinte des méthodes numériques de résolution. L'étude numérique systématique des problèmes de contrôle fera l'objet d'une publication ultérieure.

On notera que ce travail étant destiné à être lu (autant que possible) par des physiciens et des mathématiciens, on a cru bon de développer certains détails, classiques pour le spécialiste.

(1) Section Théorique des Gaz lonisés - Département de Fusion Contrôlée

C.E.A - EURATOM - Fontenay-aux-Roses 92

(2) Il y a le vide entre le plasma ef la coque 


\section{1 - DESCRIPTION DU PROBLEME}

\section{1 - LE DISPOSITIF}

Un plasma confiné est en équilibre dans une machine de type TOKOMAK [1] .

\section{COUPE DU TOKOMAK dE FONTENAY-AUX-ROSES.}

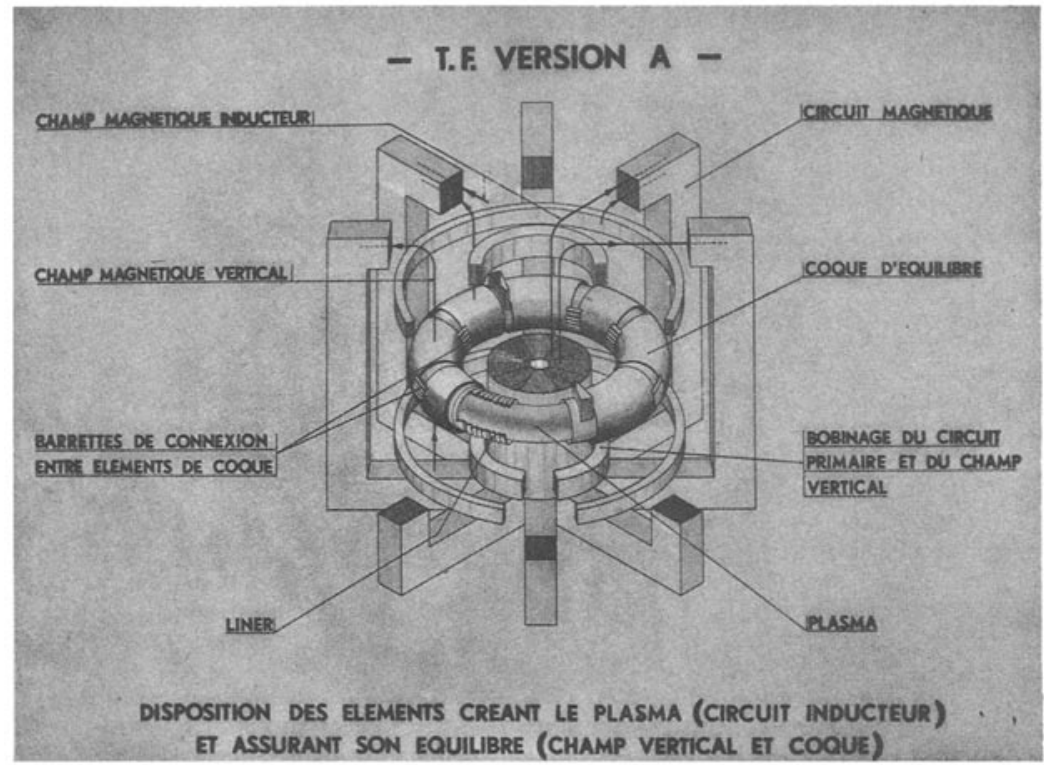

Dans cette configuration, lorsque le plasma est confiné, les lignes magnétiques s'enroulent sur sa surface. En effet, le champ magnétique crêê par le courant induit circulant le long du plasma en suivant un grand cercle, a une composante normale à la surface, nulle.

Les premiers résultats expérimentaux [2] , [3], laissent supposer que la forme de la section droite du plasma est un facteur important du chauffage ohmique. En particulier, pour la même surface de la section droite, on remarque, numériquement, que l'on peut faire passer un courant deux fois plus intense dans une forme elliptique que dans une forme circulaire. 
Le but est donc de rechercher les conditions sous lesquelles à l'équilibre le plasma a une section droite de forme donnée.

\section{FORMULATION PRATIQUE PROPOSEE pour résoudre ce problème}

. On se fixe la forme du plasma à l'équilibre

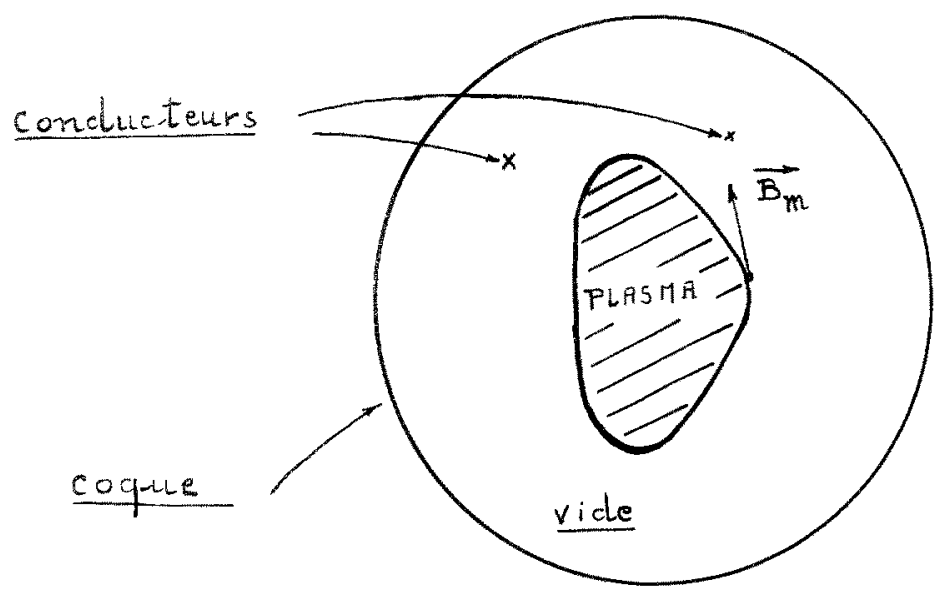

- On suppose que pour cet équilibre, on connaît la répartition du champ magnétique $\vec{B}$ meridien, créé par la décharge électrique à l'intérieur du plasma. $\vec{B}$ méridien est tangent à la surface du plasma (qui est donc une surface magnétique).

- Pour confiner le plasma à l'intérieur de la coque sous vide, on cherche à disposer les conducteurs le long de grands cercles du tore et à calculer l'intensité du courant passant : ces courants doivent créer dans la cavité coque -plasma un champ magnétique induit dont la résultante avec le champ magnétique $\vec{B}_{m}$ est la plus petite possible, en module.

Pour cela, nous avons à notre disposition trois paramètres:

$\left.1^{\circ}\right)$ - Le nombre de conducteurs $N_{c}$

$\left.2^{\circ}\right)$ - L'intensité du courant dans chacun des conducteurs, $I_{c}$

$3^{\circ}$ ) - La position des conducteurs dans la coque

Nous serons amenés à introduire une contrainte suppiémentaire d'ordre technologique.

$\sum_{i=1}^{N c}||_{c_{i}} \mid \quad$ doit être neftement inférieure à l'intensité totale $\left.\right|_{\text {total }}$ du courant de décharge à travers le plasma. 


\section{Remarque}

On ne fait aucune hypothèse sur la surface de la section droite des conducteurs.

\section{II - LES EQUATIONS DE MAXWELL}

La répartition d'un champ magnétique créé par un courant est donnée par les équations de Maxwell :

$$
\operatorname{Rot} \vec{B}=\mu_{0} \vec{J}
$$

$$
\text { Div } \vec{B}=0
$$

Etant donnée la symétrie du système, nous utiliserons des coordonnées cylindriques $(p, \varphi, z)$ où $z$ est porté par l'axe de révolution du tore. On note $\left(\overrightarrow{e_{p}}, \overrightarrow{e_{\varphi}}, \vec{e}_{z}\right)$ le trièdre orthonormé usuel en un point donné de l'espace.

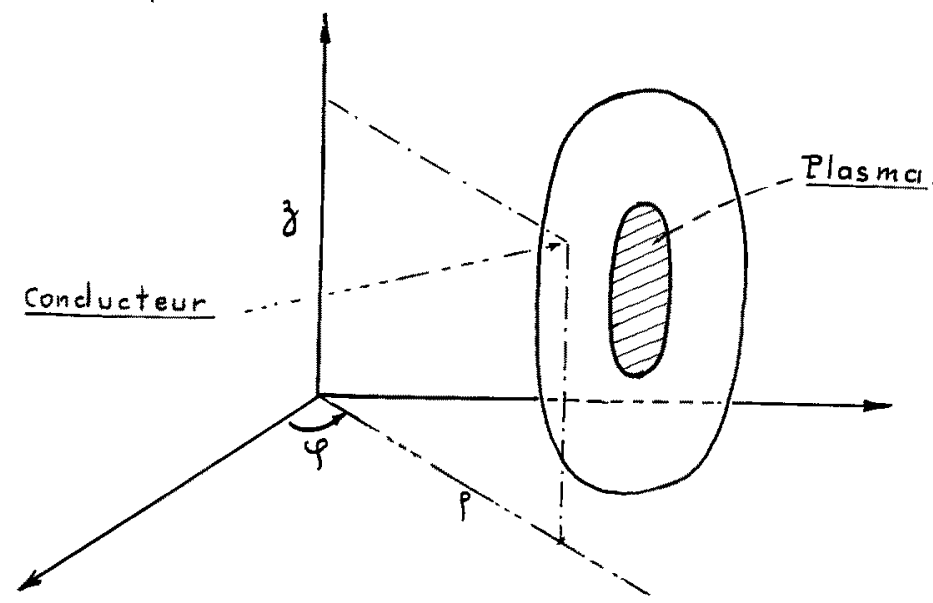

Les conducteurs étant placés le long de grands cercles, $\vec{B}$ est indépendant de la variable $\varphi$.

L'équation (1-2) s'écrit alors :

$$
\operatorname{div} \vec{B}=\frac{1}{\rho} \frac{\partial}{\partial \rho}\left(\rho B_{\rho}\right)+\frac{\partial}{\partial z} B_{z}=0
$$


Si nous posons:

$$
\begin{aligned}
& B_{z}=-\frac{1}{p} \frac{\partial F}{\partial F} \\
& B p=\frac{1}{p} \frac{\partial F}{\partial z}
\end{aligned}
$$

l'équation div $\vec{B}=0$ est identiquement vérifiée.

Supposons comme en [4] que $B_{\varphi}=\frac{f(P, z)}{P}$ et écrivons $\vec{B}$ sous la forme:

$$
\vec{B}=\frac{\rho}{\rho} \overrightarrow{e_{\varphi}}+\frac{\overrightarrow{e_{\varphi}}}{\rho} \wedge \operatorname{grad} F
$$

La quantité $\frac{\overrightarrow{e_{\varphi}}}{\rho} \wedge$ grad $F$ est le champ méridien.

\section{Remarques}

- Si l'on prend le rotationnel de $\vec{B}$, comme rot.grad $f=0$, seule reste la composante suivant $\vec{e}_{\varphi}$, donc:

$$
\overrightarrow{\operatorname{sot}} \vec{B}=\overrightarrow{e_{\varphi}}\left\{\frac{\partial B_{p}}{\partial z}-\frac{\partial B_{z}}{\partial p}\right\}=\mu_{0} \vec{J}
$$

- Nous avons indiqué que les conducteurs se trouvaient le long de grands cercles du tore, d'où:

$$
\vec{J}=\vec{e}_{p} \cdot J(p, z)
$$

Alors l'équation (1.1) s'écrit:

$$
\frac{\partial B_{p}}{\partial z}-\frac{\partial B_{z}}{\partial p}=\mu_{0} \cdot J(p, z)
$$

Si nous remplaçons $B_{p}$ ef $B_{z}$ par leurs valeurs fonction de $F$, nous obtenons l'équation:

$$
\frac{1}{p} \frac{\partial^{2} F}{\partial z^{2}}+\frac{\partial}{\partial p}\left(\frac{-1}{p} \cdot \frac{\partial F}{\partial p}\right)=\mu_{0}^{-t_{0}} \cdot J(p \cdot z)
$$


- grad $F$ représente le vecteur gradient de $F$ dans l'espace $R^{3}$; ses coordonnées dans le trièdre local $\left(\vec{e}_{p}, \vec{e}_{\varphi}, \vec{e}_{z}\right)$ s'écrivent classiquement: $\left(\frac{\partial F}{\partial \rho}, 0, \frac{\partial F}{\partial z}\right)$

La signification de l'opérateur divergence est similaire, et on rappelle que :

$$
\operatorname{div}\left(A_{p} \overrightarrow{e_{p}}+A_{\varphi} \overrightarrow{e_{\varphi}}+A_{z} \overrightarrow{e_{z}}\right)=\frac{1}{p} \cdot \frac{\partial}{\partial p}\left(p A_{p}\right)+\frac{1}{\rho} \cdot \frac{\partial}{\partial \varphi}\left(A_{\varphi}\right)+\frac{\partial A_{z}}{\partial z}
$$

Ainsi,

$$
\operatorname{div}\left(\frac{1}{\rho^{2}} \operatorname{gracl} F\right)=\frac{1}{\rho}\left\{\frac{\partial}{\partial \rho}\left(\frac{1}{\rho} \frac{\partial F}{\partial \rho}\right)+\frac{\partial}{\partial z}\left(\frac{1}{\rho} \frac{\partial F}{\partial z}\right)\right\}
$$

et l'équation (1.5) s'écrit :

$$
p \operatorname{cliv}\left(\frac{1}{p^{2}} \operatorname{grad} F\right)=\mu_{0} J(\rho, z)
$$

Sachant que :

$$
\begin{aligned}
& B_{z}=-\frac{1}{p} \cdot \frac{\partial F}{\partial p} \\
& B_{p}=\frac{1}{p} \frac{\partial F}{\partial z}
\end{aligned}
$$

\section{III - LES CONDITIONS AUX LIMITES}

Notations :

$\Omega$ est l'ouvert constitué par la couronne entre le plasma et la coque

$\Gamma_{f}$ le bord du plasma

$\Gamma_{0}$ la coque

$\Omega_{1}$ le plasma.

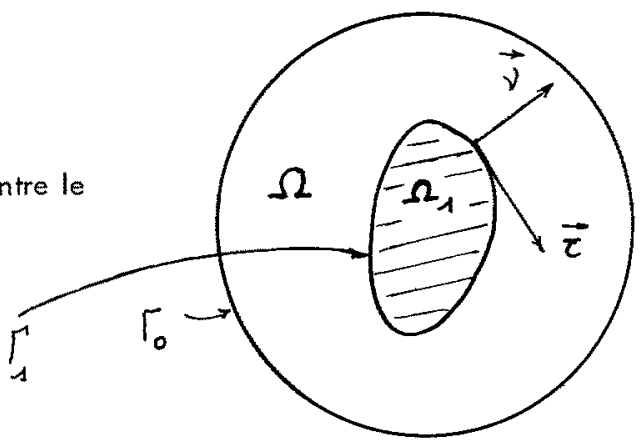


114

Le but du constructeur est diavoir sur $\Gamma_{1}$ et $\Gamma_{0}, \vec{B}_{\text {conducteur }} \cdot \vec{Y}=0$ et sur $\Gamma_{1}\left|\mathrm{~B}_{\text {conducteur }}\right|$ voisin de $\left|\overrightarrow{B_{\text {méridien }}}\right|$.

$\gamma$ étant la normale à la frontière $\Gamma_{0}$ ou $\Gamma_{1}$.

Sach ant que $\vec{\vartheta} \cdot \overrightarrow{e_{\varphi}}=0$, nous obtenons :

$$
\begin{aligned}
\vec{B} \cdot \vec{V} & =\left(\overrightarrow{e_{\varphi}} \cdot \frac{f}{\rho}+\overrightarrow{e_{\varphi}} \cdot \frac{1}{\rho} \wedge \operatorname{grad} F\right) \cdot \vec{\nu} \\
& =\left(\overrightarrow{e_{\varphi}} \cdot \frac{1}{p} \wedge \operatorname{grad} F\right) \cdot \vec{\nu} \\
& =\left(\frac{\overrightarrow{e_{\varphi}}}{\rho} \wedge \vec{\gamma}\right) \cdot \operatorname{grad} F=\frac{\vec{\tau}}{p} \cdot \operatorname{grad} F
\end{aligned}
$$

donc $\vec{B} \cdot \vec{Y}=0$ si la dérivée tangentielle $\frac{\partial F}{\partial \nu}=\vec{\tau} \cdot$ gradF est nulle.

La condition aux limires se traduit par:

Fest constant sur chacune des frontières $\Gamma_{0}$ et $\Gamma_{1}$ -

La fonction $F$ étant définie à une constante additive près, nous la prenons nulle sur $\Gamma_{0}$ et nous notons $\gamma$ sa valeur sur $\Gamma_{1}$ -

Soif: \begin{tabular}{llll|}
$(1.7)$ & $F=0$ & sur & $\Gamma_{0}$ \\
& $F=\gamma$ & sur & $\Gamma_{1}$
\end{tabular}

Xest une inconnue du problème.

IV - EN RESUME

. En chaque point s de $\Gamma$ associons le trièdre défini classiquement par $(\vec{v}, \vec{\tau}, \vec{b})$.

Dans ce repère, grad $F$ a pour composantes: $\left(\frac{\partial F}{\partial \nu}, \frac{\partial F}{\partial \tau}, \frac{\partial F}{\partial b}\right)$

De la définition de $\vec{B}$ donnée en (1.3) nous déduisons le champ magnétique sur $\Gamma_{1}$ créé par le courant dans les $I_{c}$ conducteurs soit:

(1.8) $\left.B(J)_{\text {conductour }}\right|_{\Gamma_{1}}=\frac{1}{\rho} \frac{\partial F}{\partial \nu} \mid \Gamma_{7}-$ 
- L'objectif est de construire un champ magnétique $\vec{B}_{\text {conducteur }}$ porté par la tangente $\vec{Z}$ en chaque point de la frontière $\Gamma_{1}$, et égal à $\vec{B}_{\text {méridien }}$ donné.

Le problème que l'on a à résoudre se pose donc ainsí :

Dans l'ouvert connexe $\Omega$ ci-contre calculer la fonction d'état $F$ solution de :

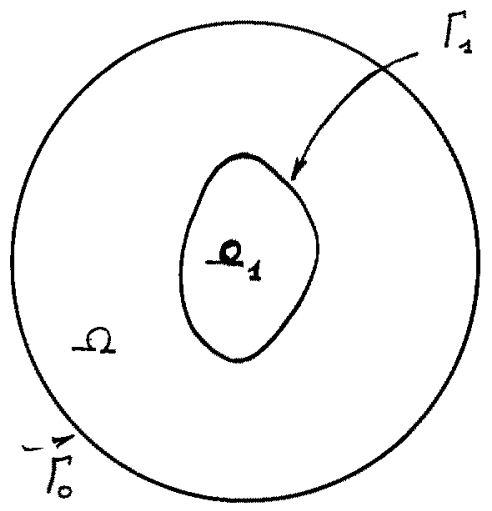

$$
\text { (1.9) }\left\{\begin{array}{l}
p \operatorname{div}\left(\frac{1}{p} \operatorname{grad} F\right)=\mu_{0} J \text { dans } \Omega \\
F=0 \quad \text { sur } \Gamma_{0} \\
F=\gamma \quad \text { sur } \Gamma_{1}
\end{array}\right.
$$

tel que $\left.\quad \frac{-1}{p} \frac{\overrightarrow{\partial F}}{\partial \nu}\right|_{\Gamma_{1}}=\vec{B}$ méridien.

Nous allons exprimer ce problème sous la forme d'un problème de contrôle:

Un ensemble de conducteurs étant fixés dans $\Omega$, rechercher le contrôle $J$ ( $J$ : intensité du courant) tel que F, solution (1.9), minimise la fonction coût, c'est à dire vérifie les contraintes suivantes:

$$
\left\{\begin{array}{l}
\left|\vec{B}_{\text {meridien }}-\vec{B}_{\text {conducteur }}\right|=\left|\vec{B}_{\text {mer. }}-\frac{1}{p} \frac{\overrightarrow{\partial F}}{\partial V}\right| \Gamma_{1} \\
\int_{\Omega}|J| d \omega, \text { nul ou le plus petit possible } \\
\text { courant total } \leqslant I_{\max } \text { défini précédemment. }
\end{array}\right.
$$

\section{Remarque 1}

On peut présenter le problème ci-dessus sous une forme équivalente, parfois numériquement plus commode. 
Nous pouvons permuter la condition aux limites sur $\Gamma_{1}$ et la première contrainte. Alors le problème s'exprime comme suit :

Chercher $J$, tel que F, solution de:

(1.11)

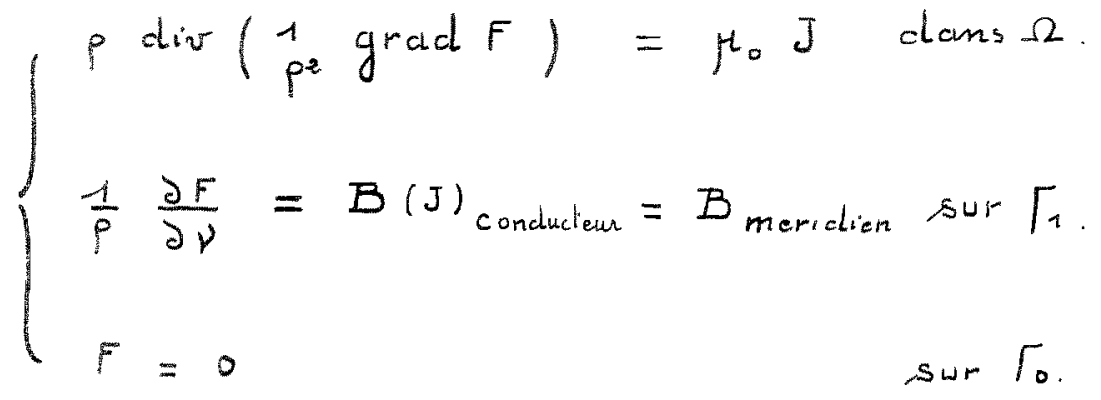

Vérifie les conditions suivantes:

(1. 12)

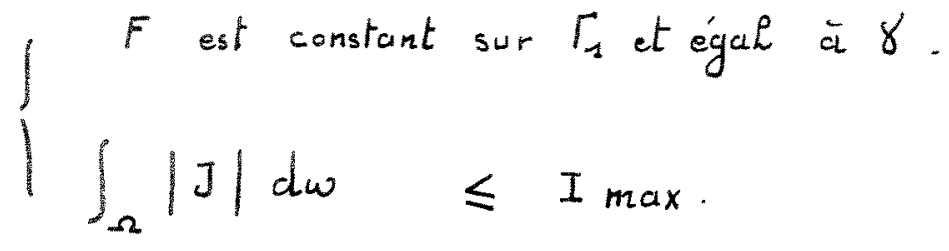

\section{Remarque 11}

De la façon dont est défini le courant $J$ dans les problèmes ci-dessus, on constate que les conducteurs occupent tout l'espace compris entre le plasma ef la coque.

Si l'on est amené à éliminer des conducteurs dans une zone, cela ne change rien mathématiquement au problème. Il suffit d'imposer que le courant est nul dans cette zone. 


\section{1 - ETUDE DES EQUATIONS DU SYSTEME}

Les équations régissant l'état du système sont essentiellement les équations (1.9) dont nous nous proposons de préciser le sens et la formulation.

Une section droite du tore est rapportée auxaxes $O_{p}, O_{z}$, où $O_{z}$ désigne l'axe du tore.

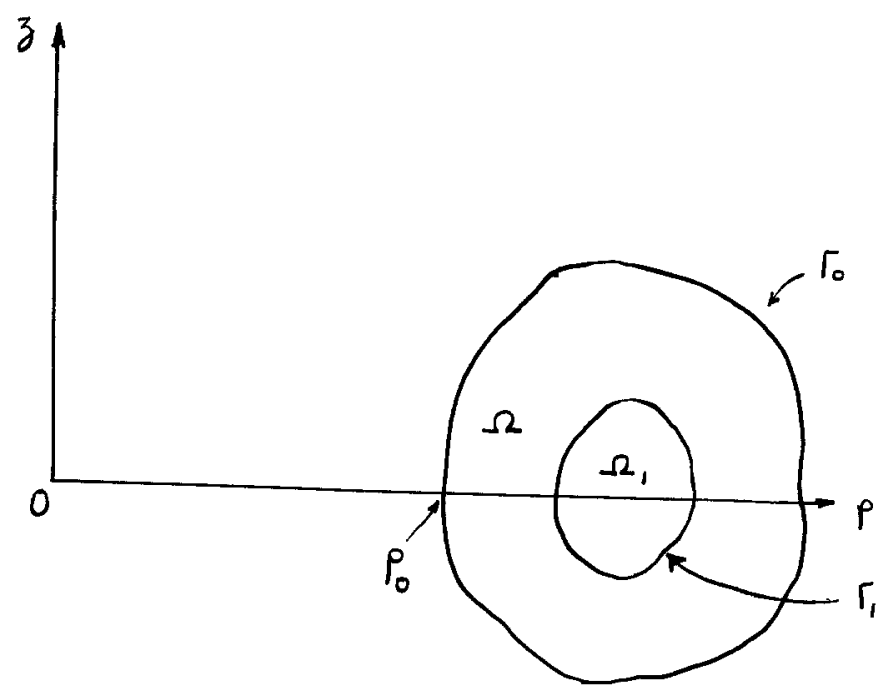

Le plasma occupe le domaine (connexe) $\Omega$ de frontière $\Gamma_{1}$, la coque a pour frontière $\Gamma_{0}$, et le domaine $\Omega$ compris entre $\Gamma_{0}$ et $\Gamma_{1}$ est vide à l'exception des conducteurs électriques dont l'implantation nous intéresse particulièrement. On observera donc que :

$$
\rho_{1} \rightleftharpoons \rho \geqslant \rho_{0}>0 \quad \text { dans } \bar{\Omega} \cup \bar{\Omega}_{1}
$$

Les équations d'état (I.9) sont en fait à compléter par la condition suivante qui exprime que la circulation de $\vec{B}$ le long de $\Gamma_{1}$ est égale à l'intensité totale I du courant dans le plasma (I est connu $\left.=\int_{\Gamma_{1}}\left|B_{m}\right| d l\right):(1)$

$$
\int_{\Gamma_{1}} \vec{B}_{\text {vide }} \cdot \vec{\tau} d l=\int_{\Gamma_{1}} \vec{B}_{\text {plosmal }} \cdot \vec{\tau} d l=\int_{\Omega_{1}} \mu_{0} J_{p} d x=I
$$


soit en serme de F :

$$
\int_{\Gamma_{1}} \frac{1}{P} \frac{\partial F}{\partial \nu} d P=I
$$

Pour donner une formulation variationnelle de (1.9) (11.2) on introduit l'espace de Sobolev:

$$
H^{2}(\Omega)=\left\{v \in L^{2}(\Omega), \frac{\partial v}{\partial p}, \frac{\partial v}{\partial z} \in L^{2}(\Omega)\right\}
$$

qui est de Hilbert pour le produit scalaire:

(II.3) $((u, v))=(u, v)+\left(\frac{\partial u}{\partial \rho}, \frac{\partial v}{\partial \rho}\right)+\left(\frac{\partial u}{\partial z}, \frac{\partial v}{\partial z}\right)$,

où

$$
(f, g)=\int_{\Omega} f(p, z) \cdot g(p, z) \cdot d \rho \cdot d z .
$$

On considère le sous espace $V$ de $H^{\prime}(\Omega)$ formé des fonctions nulles sur $\Gamma_{0}$ et qui sont constantes sur $\Gamma_{1}$ (au sens des théorèmes de trace, of. Lions-Magenes [1]); $V$ est fermé dans $H^{1}(\Omega)$, c'est un sous espace hilbertien de $H^{1}(\Omega)$.

On définit aussi sur $V$ le produit scalaire :

$$
[\mu, v]=\int_{\Omega} g r a d-u \cdot g r a d v \frac{d p \cdot d z}{\rho},
$$

où

$$
\text { gradu }=\nabla_{\mu}=\left\{\frac{\partial u}{\partial p}, \frac{\partial u}{\partial z}\right\}
$$

On vérifie que $\llbracket$, $]$ est un produit scalaire continu sur $Y$. Par ailleurs en vertu de $(I I .1)$ et de l'inégalité de Poincaré,

$$
\| \cdot u]^{2} \geqslant \frac{1}{p_{1}} \int_{\Omega}|g \mathrm{rad} \cdot u|^{2} d p \cdot d z \geqslant \frac{c(\Omega)}{p_{0}} \int_{\Omega}-u^{2} d p d z
$$


Ainsi (II.4) est un produit scalaire hilbertien sur V et la norme associée \, „ est équivalente à la norme $\|\cdot\|-$

Soit GEV; multipliant (1.10) par $G$ et intégrant sur $\Omega$ on trouve :

(11.5) $-\int_{\Omega} \frac{1}{p}$ grad $F \cdot$ grad $G d p \cdot d z+\int_{\Gamma_{1}} \frac{1}{p} \frac{\partial F}{\partial \nu} \cdot G d l=\mu_{0} \int_{\Omega} J \cdot G \cdot d p \cdot d z$.

Utilisant $(11.2)$ on voit alors que $F$ est solution de $(1.10)(11.2)$ si et seulement si FEV et vérifie :

$$
\llbracket F, G \rrbracket=-\mu_{0} \int_{\Omega} J \cdot G \cdot d \rho \cdot d z+I \cdot \int_{\Gamma_{1}} G d L .
$$

Utilisant le lemme de Lax Milgram, on voit alors que pour tout $\mathrm{J}$ donné dans $\mathrm{L}^{2}(\Omega)$ il existe un $F$ unique dans $V, F=F(J)$ qui soit solution de (11.5) (ou (1.10)(11.4)).

Nous rappelons la terminologie usuelle en contrôle optimal,

- J est le contrôle

. $F=F(J)$ solution de (II.5) est la fonction décrivant l'état du système.

\section{II - FORMULATION DES DIFFERENTS PROBLEMES DE CONTROLE}

Rappelons que le but poursuivi est la recherche de courants J répartis dans $\Omega$, tels que la dépense de courant ne soit pas excessive et que, autant que possible:

$$
\frac{1}{\rho} \cdot \frac{\partial F}{\partial \nu}=B_{m}(\text { domé }) \text { sur } \Gamma_{1}
$$

On peut mesurer le coùt du courant soit par son intensité totale, $\int_{\Omega}|J| d l . d z$ soit par son énergie $\int_{\Omega} J^{2} d p d z$. Nous optons évidemment pour la seconde expression mathématiquement prêférable. Nous posons alors les problèmes suivants :

Problème de Contrôle $\left(P_{\lambda}\right)$ Méthode de pénalisation.

Soit $\lambda>0$ fixé. On cherche $J \in L^{2}(\Omega)$ qui réalise le minimum de :

$$
\frac{1}{\lambda} \int_{\Gamma_{1}}\left(\frac{1}{\rho} \frac{\partial F}{\partial \nu}-B_{m}\right)^{2} d \rho+\int_{\Omega} J^{z} d \rho d z
$$


où $\mathrm{F}=\mathrm{F}(J)$.

Problème de Contrôle $\left(P_{0}\right)$

On cherche $\mathrm{J} \epsilon \mathrm{L}^{2}(\Omega)$, qui réalise le minimum de :

$$
\int_{\Omega} J^{2} d p d z
$$

parmi toutes les fonctions 3 telles que:

$$
\frac{\alpha}{p} \frac{\partial F(J)}{\partial \nu}=B_{m} \text { sur } \Gamma_{A}
$$

On a les résultầs suivants :

Théorème 11.1

(i) Pour tout $\lambda>0$ fixé, le problème $P \lambda$ possède une solution unique définie par le contrôle $\overline{J_{\lambda}}$ et l'état $\bar{F}_{\lambda}$

(ii) Le problème $\left(P_{0}\right)$ possède une solution unique $\left(\right.$ contrôle $\bar{J}_{0}$, êtat $\bar{F}_{0}$ )

(iii) Lorsque $\lambda \rightarrow 0$.

$$
\bar{J}_{\lambda} \rightarrow \bar{J}_{0} \operatorname{dans} L^{2}(\Omega) ; \bar{F}_{\lambda} \rightarrow \bar{F}_{0} \text { dans } H^{1}(\Omega)
$$

\section{Démonstration}

(i) Par application des théorèmes de régularité pour les problèmes elliptiques (cf. Agmon-DouglisNiremberg [1], Lions-Magenes [1]), on voit que I'application affine.

$$
J \longrightarrow F=F(J)
$$

est continue de $L^{2}(\Omega)$ dans $H^{2}(\Omega)$. Par application des théorèmes de traces, I'application

$$
F \longrightarrow \frac{1}{p} \frac{\partial F}{\partial y} \mid \Gamma_{1}
$$

est continue de $H^{2}(\Omega)$ dans $H \frac{1}{2}\left(\Gamma_{1}\right)$ et donc $L^{2}\left(\Gamma_{1}\right)$. L'application

$$
\left.J \rightarrow \frac{1}{p} \frac{\partial F}{\partial \nu}\right|_{\Gamma_{1}}
$$


est done continue de $L^{2}(\Omega)$ dans $L^{2}\left(\Gamma_{1}\right)$.

Il en résulte aisément que la fonction coût en (II.8) est convexe continue en $\rfloor$; elle est aussi strictement convexe, et tend vers $+\infty$ lorsque $|J|_{L^{2}(\overrightarrow{\Omega)}}$ : Elle atteint donc son minimum sur $L^{2}(\Omega)$ en un point unique, $\overline{J_{\lambda}}$. (cf. J.L. Lions $[3]$ ). On note $\overline{F_{\lambda}}$ la solution correspondante de (II.5).

(ii) L'ensemble des contrôles admissibles pour $\left(P_{0}\right)$ est l'ensemble des $J \in L^{2}(\Omega)$ tels que (II.10) soit vérifié : cet ensemble n'est pas vide. En effet il existe des fonctions $F$ dans $H^{2}(\Omega)$ telles que :

$$
\left.F\right|_{\Gamma_{1}}=1 ;\left.\quad \frac{\partial F}{\partial \nu}\right|_{\Gamma_{1}}=\rho B_{m}
$$

Cette fonction $F$ vérifie la condition

$$
\int_{\Gamma_{1}} \frac{1}{\rho} \frac{\partial F}{\partial P} d \mathcal{L}=I
$$

d'après la définition de I (cf. note ${ }^{(1)}$ p.10). Soit à présent $\theta$ une fonction $\mathcal{E}^{\infty}$ sur $\bar{\Omega}$, égale à 1 dans un voisinage de $\Gamma_{1}$ ef à 0 dans un voisinage de $\Gamma_{0}$. On vérifie aisément que $\theta F$ est un étał admissible ef que :

$$
J=\frac{1}{H_{0}} \operatorname{div} \frac{1}{\rho} \operatorname{grad}(\theta F) \text {. }
$$

est un contrôle admissible pour $\left(P_{0}\right)^{(1)}$

L'ensemble des contrôles admissibles est convexe fermé d'après (11. 12). On démontre comme en (i) l'existence ef l'unicité d'un contrôle optimal ici noté $\bar{J}_{0}$ (cf. Lions $[3]$ ); $\bar{F}_{0}$ est la solution de (II.5) pour $\mathrm{J}=\overline{\mathrm{J}}_{\mathrm{0}}$.

(iii) Par définition de $\bar{J}_{\lambda}$,

(II.13) $\frac{1}{\lambda} \int_{\Gamma_{1}}\left(\frac{1}{p} \cdot \frac{\partial F_{\lambda}}{\partial y}-B_{m}\right)^{2} d l+\int_{\Omega}\left(\overline{J_{\lambda}}\right)^{2} d \rho d z \leqslant \int_{\Omega}\left|\bar{J}_{0}\right|^{2} d p d z$.

Cela montre que la suite $\bar{J}_{\lambda}$ est bornée dans $L^{2}(\Omega)$ lorsque $\lambda \rightarrow 0$, et que :

$$
\int_{\Gamma_{1}}\left(\frac{1}{\rho} \frac{\partial F_{\lambda}}{\partial \varphi}-B_{m}\right)^{2} d L \leqslant c \lambda
$$

\footnotetext{
$\overline{(1)}$ L'existence de contrôles admissibles pour $\left(P_{0}\right)$ résulte aussi de (iii)
} 
II existe donc une suite $\lambda_{i} \rightarrow 0$, telle que $\bar{J}_{\lambda_{i}} \rightarrow J_{*}$ dans $L^{2}(\Omega)$ faible; d'après $(11.11)$, (II. 12)

$$
\overline{F X_{i} \longrightarrow F\left(J_{*}\right)}
$$

dans $H^{2}(\Omega)$ faible, ef

$$
\frac{1}{p} \cdot \frac{\partial \bar{F}_{\lambda_{i}}}{\partial \eta} \longrightarrow \frac{1}{\rho} \cdot \frac{\partial F\left(J_{*}\right)}{\partial y}
$$

dans $L^{2}\left(\Gamma_{1}\right)$ faible. Daprès $(11.14)$

$$
\frac{1}{p} \cdot \frac{\partial F\left(J_{*}\right)}{\partial \nu}=B_{m}
$$

ce qui montre que $J_{*}$ esi un contrôle admissible pour $\left(P_{0}\right)$. Par ailleurs, d'après (11.13):

$$
\begin{aligned}
& \int_{\Omega}\left(J_{*}\right)^{2} d \rho d z \leqslant \lim _{\lambda_{i} \rightarrow 0} \int_{\Omega}\left(\bar{J}_{\lambda_{i}}\right)^{2} d \rho d z \\
& \leqslant \lim _{\lambda_{i} \rightarrow 0} \sup _{\Omega}\left(J_{\lambda_{i}}\right)^{2} d \rho d z \leqslant \int_{\Omega}\left(\bar{J}_{0}\right)^{2} d \rho d z
\end{aligned}
$$

Ainsi $J_{*}$ est contrôle optimale pour $\left(P_{0}\right)$ et par lunicité, $J_{*}=\bar{J}_{0}$. On vérifie encore que la suite $\bar{J}_{\lambda}$ tout entière converge vers $\bar{J}_{0}$ dans $L^{2}(\Omega)$ faible (raisonner par l'absurde). Utilisant encore (II.15) (avec $J_{*}=\bar{J}_{0}$ ), on voit que :

$$
\int_{\Omega}\left(\bar{J}_{\lambda}\right)^{2} d \rho \cdot d z-\int_{\Omega}\left(\bar{J}_{0}\right)^{2} d \rho d z
$$

ce qui montre que :

$$
\bar{J}_{\lambda} \rightarrow \bar{J}_{0} \text { dans } L^{2}(\Omega) \text { fort. }
$$

Ensuite (11.11) donne:

$$
\bar{F}_{\lambda \longrightarrow \overline{F_{0}}} \quad \text { dans } H^{2}(\Omega) \text { Fort. }
$$

La démonstration du thêrème est achevée. 
Remarque 11.1

Le contrôle optimal peut-être caractérisé par les relations usuelles d'extrémalité faisant interveni l'état ef l'état adjoint; cf. Boujot-Morera-Temam [4] où l'on trouvera l'étude numérique des problèmes de contrôle précédents. 


\section{II) - AUTRE PROBLEMES DE CONTROLE}

\section{LA METHODE DES MOINDRES CARRES}

Nous voulons développer ici une approche différente du problème qui est moins classique du point de vue de la théorie du contrôle, mais qui permet de mettre l'accent sur certaines quantités utiles en physique.

L'étude qui suit est limitée au cas où les courants circulent dans des conducteurs ponctuels dont le nombre $N_{c}$ ef la position $\left(\varphi_{i}, z_{i}\right), i=1, \ldots . N_{c}$, sont fixés à priori.

\section{1 - UN AUTRE CHOIX DES EQUATIONS D'ETAT}

Comme il a êté indiqué à la fin de la section l, on peut supposer que : $\frac{1}{\rho} \frac{\partial F}{\partial \nu}=B_{m}$. est exactement vérifiée sur $\Gamma_{1}$ et chercher à réaliser au mieux ou exactement la condition $F=$ constante sur $\Gamma_{1}$.

Pour préciser (1.11), on introduit l'espace :

$$
W=\left\{v \in H^{-1}(\Omega) \mid v=0 \text { sur } \Gamma_{0}\right\}
$$

qui est un sous espace hilbertien de $H^{1}(\Omega)$, et qui est aussi de Hilbert pour le produit scalaire

$$
\text { 【. }
$$

Remplaçant F par $\Phi$; on voit que le problème (1.11) est équivalent au problème variationel.

(III. 1) Trouver $\bar{\Phi}=\Phi(J)$ dans $W$ tel que

$$
[\Phi, \Psi]=-\int_{\Omega} \mu_{0} J \cdot \Psi d p \cdot d z+\int_{\Gamma_{1}} B_{m} \cdot \Psi d P, \forall \Psi \in W
$$

L'existence et l'unicité de $\Phi$ découle donc du lemme de Lax-Milgram.

Dans cette nouvelle approche $J$ et $\Phi(J)$ constituent le contrôle el l'état associé ; (1.11) et (1II.2) constituent les équations d'état.

\section{II - UTILISATION D'UN DEVELOPPEMENT EN SERIE DE FOURIER}

On considère une suite de fonctions $W_{n}$ définies sur la frontière $\Gamma_{1}$, qui sont linéairement indépendantes et dont les combinaisons linéaires finies sont denses dans $L^{2}\left(\Gamma_{1}\right)$. On prendra par exemple dans les applications les fonctions : 
125

(III.3) $w_{n}=w_{n}(s)=\cos 2 n \pi \frac{s}{L}, \quad n=0,1, \ldots$

où s désigne l'abscisse curviligne sur $\Gamma_{1}$, et $L$ la longueur totale de $\Gamma_{1}$.

Pour tout $n$, on appelle $v_{n}$ la solution du problème mixte.

(III.4)

$$
\left\{\begin{array}{l}
\rho \operatorname{div}\left(\frac{1}{p^{*}} \operatorname{grad} v_{n}\right)=0 \text { dans } \Omega, \\
v_{n}=0 \operatorname{sur} \Gamma_{0}, \\
\frac{1}{\rho} \frac{\partial v_{n}}{\partial \varphi}=w_{n} \operatorname{sur} \Gamma_{1} .
\end{array}\right.
$$

Le problème (III.4) est équivalent au problème variationnel (III.2) dans lequel $J=0$ et $B_{m}=W_{n}$ :

(III.5) Trouver $V_{n} \in W$ tel que

$$
\llbracket v_{n}, v \rrbracket=\int_{\Gamma_{1}} w_{n} \cdot v d L, \forall v \in W
$$

L'existence ef l'unicité de $v_{n}$ découle encore du lemme de Lax Milgram.

On appelle $\varphi_{n}$ la trace sur $\Gamma_{1}$ de $v_{n}$. On a le:

Lemme III. I

(i) L'espace fermé engendré par les fonctions $v_{n}$, est l'orthogonal de $H_{0}^{1}(\Omega)$ dans W muni du

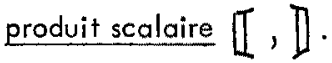

(ii) Les fonctions $\varphi_{n}=v_{n} \mid \Gamma_{1}$ sont linéairement indépendantes et forment une partie totale de $L^{2}\left(\Gamma_{1}\right)$.

Démonstration

(i) Considérons une fonction $v$ de $W$ telle que :

$$
\llbracket v, v_{n} \rrbracket=0, \forall n
$$


126

Alors, d'après (IV.3),

$$
\int_{\Gamma_{1}} v_{n} \cdot v d P=0 \quad \forall n
$$

d'après la définition des $v_{n}, v$ est nul sur $\Gamma_{1}$ et comme $v=0$ sur $\Gamma_{0}$, on a bien $v \in H_{0}^{-1}(\Omega)$

(ii) Supposons que $\sum_{n=1}^{m} \lambda_{n} \cdot \varphi_{n}=0$. Alors la fonction $v^{*}=\sum_{n=1}^{m} \lambda_{n} \cdot v_{n}$ vérifie d'après (III.2),

(111.6) $\left\{\begin{array}{l}\operatorname{div}\left(\frac{1}{\rho^{2}} \operatorname{grad} v^{*}\right)=0 . \\ v^{*}=0 \text { sur } \Gamma_{0} \text { et } \Gamma_{1} .\end{array}\right.$

ce qui entraine que $V^{*} \equiv 0$, et

$$
\left.\frac{1}{\rho} \cdot \frac{\partial v^{*}}{\partial \nu}\right|_{\Gamma_{1}}=\left.\sum_{n=1}^{m} \frac{\partial n}{\rho} \cdot \frac{\partial v_{n}}{\partial \nu}\right|_{\Gamma_{1}}=\sum_{n=1}^{m} \lambda_{n} v_{n}=0
$$

Comme les $v_{n}$ sont indépendanis, $\lambda_{1}=\ldots=\lambda_{m}=0$, et les $\varphi_{n}$ sont donc aussi indépendants.

11 résuite enfin du point (i) que les combinaisons linéaires des $\varphi_{n}$ sont denses dans $H^{1 / 2}\left(\Gamma_{1}\right)$ (= espace des traces sur $\Gamma_{1}$ des fonctions de $W$ ), et elles sont donc denses dans $L^{2}\left(\Gamma_{1}\right.$ ).

Faisons à présent $\Psi=v_{n}$ dans (III.2), et $v=\Phi$ dans (III.5). II vient

(III.7)

$$
\llbracket \phi, v_{n} \rrbracket=-\int_{\Omega} \mu_{0} J v_{n} d \rho \cdot d z+\int_{\Gamma_{1}} B_{m} \cdot v_{n} d R .
$$

(III. 8)

$$
\left[v_{n}, \phi\right]=\int_{\Gamma_{1}} w_{n} \Phi d l=\int_{\Gamma_{1}} \Phi W_{n} d l .
$$


Si la fonction $\Phi$ est constante sur $\Gamma_{1}$ (comme on le souhaite), $\Phi \equiv \gamma$ sur $\Gamma_{1}$, alors la dernière relation s'écrit :

$$
\left.\llbracket v_{n}, \Phi\right]=\gamma \int_{\Gamma_{1}} w_{n} d R
$$

Par comparaison de (III.7) et (III.9) on tire :

(III. 10)

$$
\int_{\Gamma_{1}} B_{m} \cdot v_{n} d L-\gamma \int_{\Gamma_{1}} w_{n} d l=\mu_{0} \int_{\Omega} J v_{n} d \omega, \forall n .
$$

Supposons réciproquement qu'il existe un nombre réel $\gamma$ tel que les conditions (III. 10) soient vérifiées pour tout $n$. Alors utilisant $(111.7)$ et (III.8) on voit que :

$$
\int_{\Gamma_{1}} \Phi w_{n} d L=\int_{\Gamma_{1}} \gamma w_{n} d L, \forall n .
$$

En raison des propriétés des $W_{n}$ cela implique:

$$
\left.\Phi\right|_{\Gamma_{1}}=\gamma
$$

Ainsi les relations (III.10) sont, au sens précédent équivalentes à (III.11).

\section{III - CAS DIUN NOMBRE FINI DE CONDUCTEURS}

On suppose comme indiqué au début de cette section que le courant $J$ est réparti entre $N_{c}$ conducteurs ponctuels placês en des points $\left(f_{i}, \xi_{i}\right)$ fixés. L'inconnue (le contrôle) est l'intensi té $I_{\alpha}$ parcourant le $\alpha^{\text {ième }}$ conducteur, $\alpha=1, \ldots N_{c} ;$

La fonction $\Phi$ peut encore être définie mais plus sous la forme variationnelle (III.2). On utilise à nouveau (1.11) qui devient :

$$
\text { (III.12) }\left\{\begin{array}{l}
\rho \operatorname{din}\left(\frac{1}{\rho^{2}} \operatorname{grad} \Phi\right)=\mu_{0} \sum_{\alpha=1}^{N_{c}} I_{\alpha} \delta\left(\rho-\rho_{\alpha}\right) \cdot \delta\left(z-z_{\alpha}\right) \text { dans } \Omega, \\
\frac{1}{\rho} \partial \phi=B_{m} . \\
\Phi=0 .
\end{array}\right.
$$


L'existence et l'unicité de $\Phi$ résulte par exemple de Lions-Magenes [1]; $\Phi$ est analytique dans $\Omega$ en dehors des points $\left(\rho_{\alpha}, z_{\alpha}\right)$.

Si nous supposons les fonctions $W_{n}$ continues sur $\Gamma_{1}$, les fonctions $V_{n}$ seront continues sur $\Omega$, ef les relations $(111,10)$ seront valables.

La condition $\$=\gamma$ sur $\Gamma_{1}$ sera réalisée si et seulement si

$$
\int_{\Gamma_{1}} B_{m} \cdot v_{n} \cdot d l-\gamma \int_{\Gamma_{1}} v_{n} d l=\mu_{0} \sum_{\alpha=1}^{N_{c}} I_{\alpha} v_{n}\left(\rho_{\alpha}, z_{\alpha}\right), \forall n
$$

Ainsi les relations $(111,13)$ se présentent comme un système linêaire infini pour les $N_{C}+1$ inconnues que sont $X, I_{1}, \ldots, I_{N_{c}}$.

On peut se limiter aux $N$ premières relations (III, 13), ce qui signifie que I'on remplace (III. 11) par :

$$
\int_{r_{1}}(\Phi-\gamma) w_{n} d L=0, n=1, \ldots N
$$

Les équations (111.13) pour $n=1, \ldots ; N$, constituent un système linéaire surabondant pour les inconnues $\gamma, I_{1}, \ldots, I_{N_{c}}\left(N>N_{c}+1\right)$ et on propose de résoudre le système par une méthode de moindre carrés. Cela revient à minimiser la fonctionnelle :

(III.15) $\sum_{n=1}^{N}\left\{\int_{\Gamma_{1}} B_{m} v_{n} d L-\gamma \int_{\Gamma_{1}} w_{n} d \rho-\mu_{0} \sum_{\alpha=1}^{N_{c}} I_{\alpha} v_{n}\left(\rho_{\alpha}, z_{\alpha}\right)\right\}^{2}$

II s'agit donc d'un problème de contrôle optimal beaucoup plus rudimentaire que les problèmes envisagés dans la section 11 . Toutefois le problème considêrê ici fournit des informations très utiles sur les harmoniques de la solution optimale. 


\section{IV - RESOLUTION NUMERIQUE}

\section{ORIENTATION}

Nous signalons ici la résolution numérique du problème de contrôle décrit dans III.

Pour résoudre la suite des $N$ problèmes.

$$
\left\{\begin{array}{l}
\operatorname{div}\left(\frac{1}{\rho^{2}} \operatorname{grad} F\right)=0 \quad \text { dans } \Omega \\
F \mid \Gamma_{0}=0 \\
\frac{1}{\rho} \frac{\partial F}{\partial \nu}=w_{n} \mid \Gamma_{1}, w_{n}=\cos 2 \pi n \frac{\rho}{L}, n=0, \ldots N-1
\end{array}\right.
$$

Nous avons utilisé un programme d'éléments finis dû à Terrine et Meurant

Nous donnons quelques exemples pour une configuration droite donnée et une décharge fixée dans le plasma.

Sur chaque page nous avons:

- Le schéma indiquant la position des conducteurs,

- La courbe de B méridien,

- Les différentes approximations de B méridien,

- La valeur des intensités dans chacun des conducteurs.

L'étude numérique des problèmes de contrôle décrits en II fera l'objet d'un travail ultérieur.

Toutefois, de nombreuses techniques numériques développées ci-après (implémentation de la méthode des éléments finis, calcul numérique de certaines intégrales de surface, ...) constituent déjà une partie significative des travaux numériques ultérieurs. 


\section{QUELQUES RESULTATS NUMERIQUES}

On donne les caractéristiques de l"expérience numérique simulée:

$$
\begin{array}{ll}
\text { Le Tokomak est un tore de grand rayon } & R=130 \mathrm{~cm} \\
\text { Le plasma a une section droite elliptique } & \\
\text { Cetfe ellipse a pour caractéristiques } & a=18 \mathrm{~cm} \\
& b=10 \mathrm{~cm}
\end{array}
$$

La décharge électrique dans le plasma

est de

$$
\text { - } 100 \text { kilo Ampères }
$$

La répartition du champ méridien (donné) a été calculée dans cette ellipse suivant une formule érablie par MASCHKE.

Le $\vec{B}$ méridien est représenté sur les courbes suivantes. L'origine sur la frontière $\Gamma_{1}$ a été choisie comme indiqué dans le schéma ci-dessous:

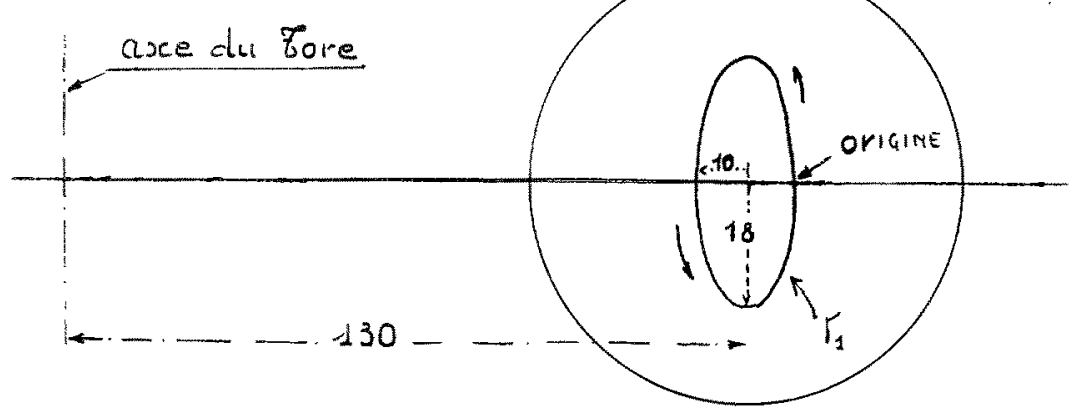

Sur chaque feville nous donnons la triangulation choisie, la place des conducteurs, avec leurs numéros. $\overrightarrow{\mathrm{Bm}}$ est calculé avec quatre fonctions de bases.

Pour chaque conducteur, la valeur de l'intensité est en kilo Ampère. 


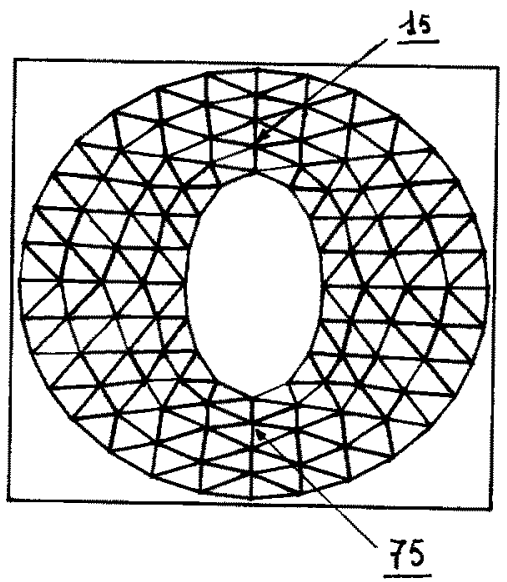

Vabeur des imiensiles dans les concludieurs consideres:

$$
\begin{aligned}
& I_{15}=-14 \mathrm{k} \cdot A_{M P} \\
& I_{75}=-14 \mathrm{k} \cdot \mathrm{AMP}
\end{aligned}
$$

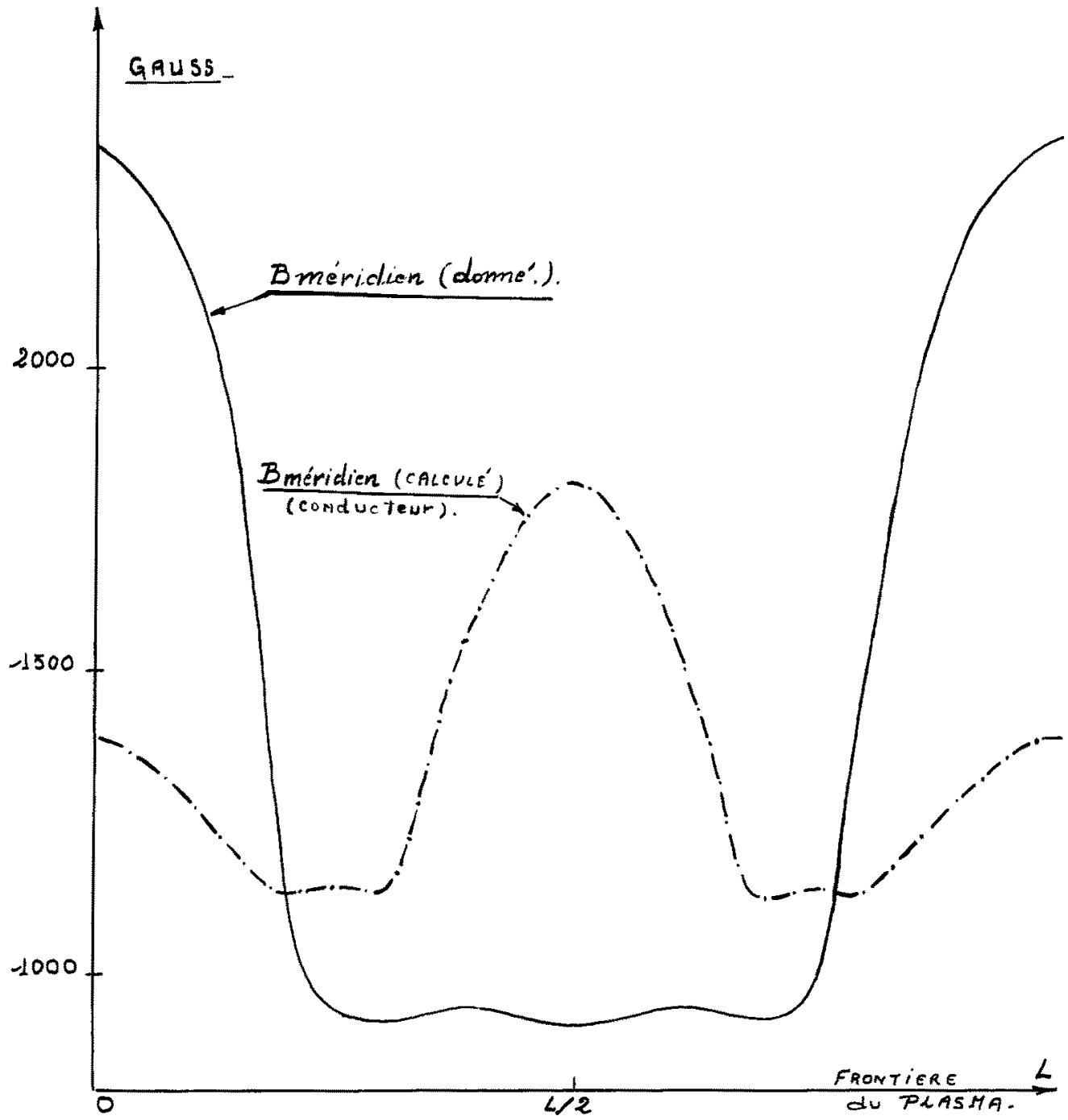




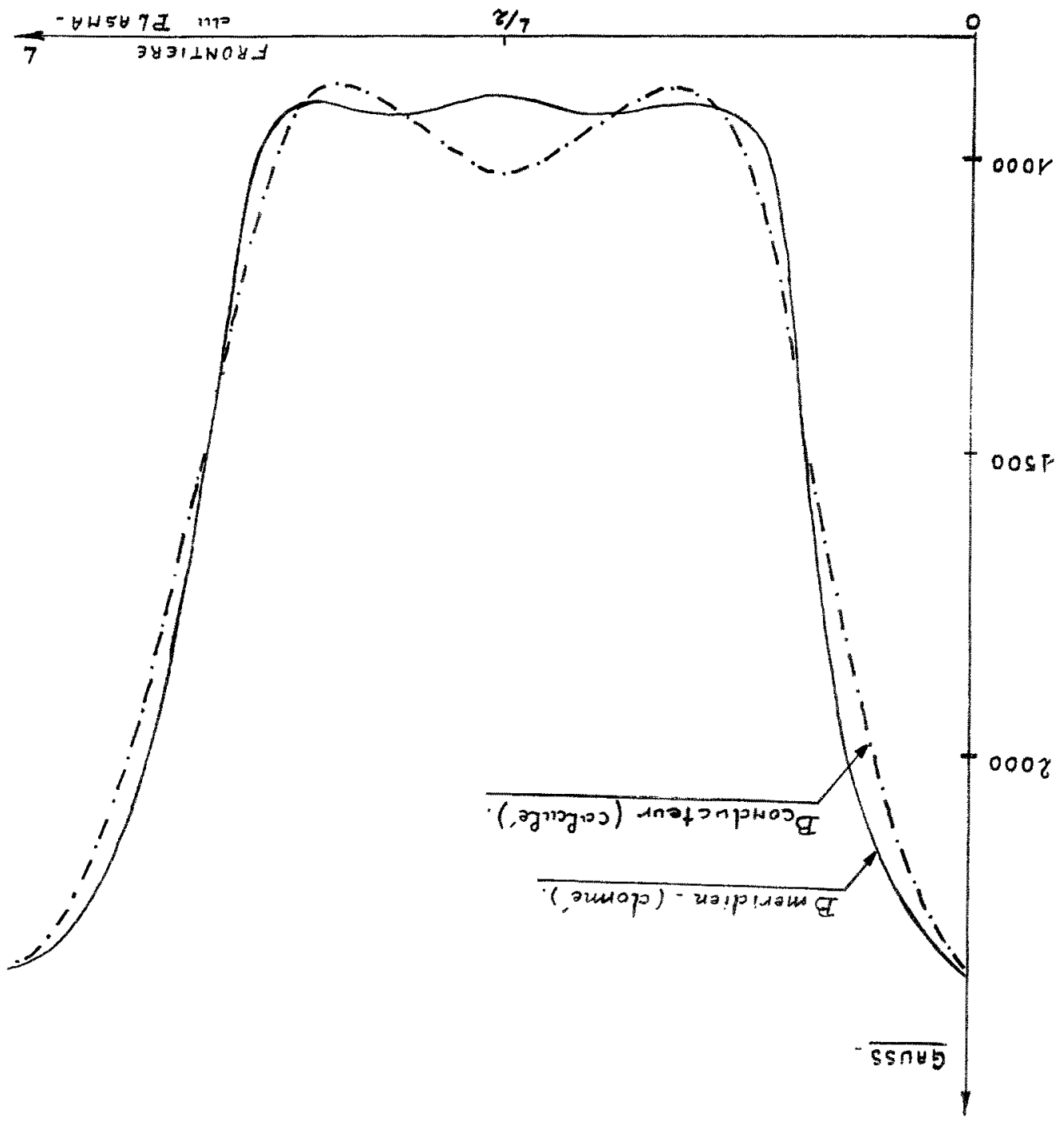

$$
\begin{aligned}
& \text { dw日. } t^{*} \varepsilon-=\& \sigma_{I} \\
& \text { JWE. } 7 t=0 \% I
\end{aligned}
$$

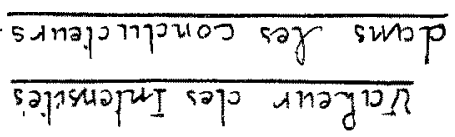

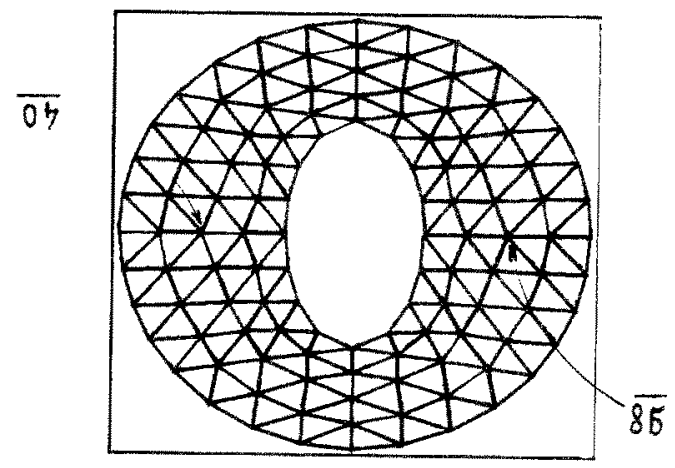



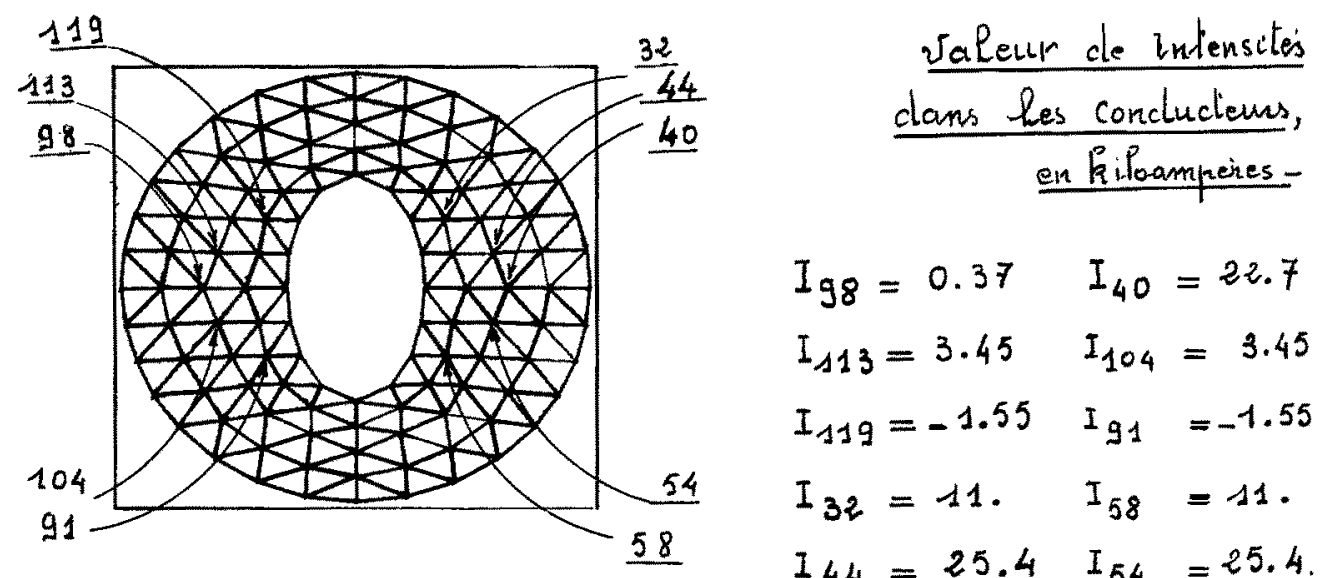

$$
\begin{array}{ll}
I_{98}=0.37 & I_{40}=22.7 \\
I_{113}=3.45 & I_{104}=3.45 \\
I_{119}=-1.55 & I_{91}=-1.55 \\
I_{32}=11 . & I_{58}=11 . \\
I_{44}=25.4 & I_{54}=25.4 .
\end{array}
$$

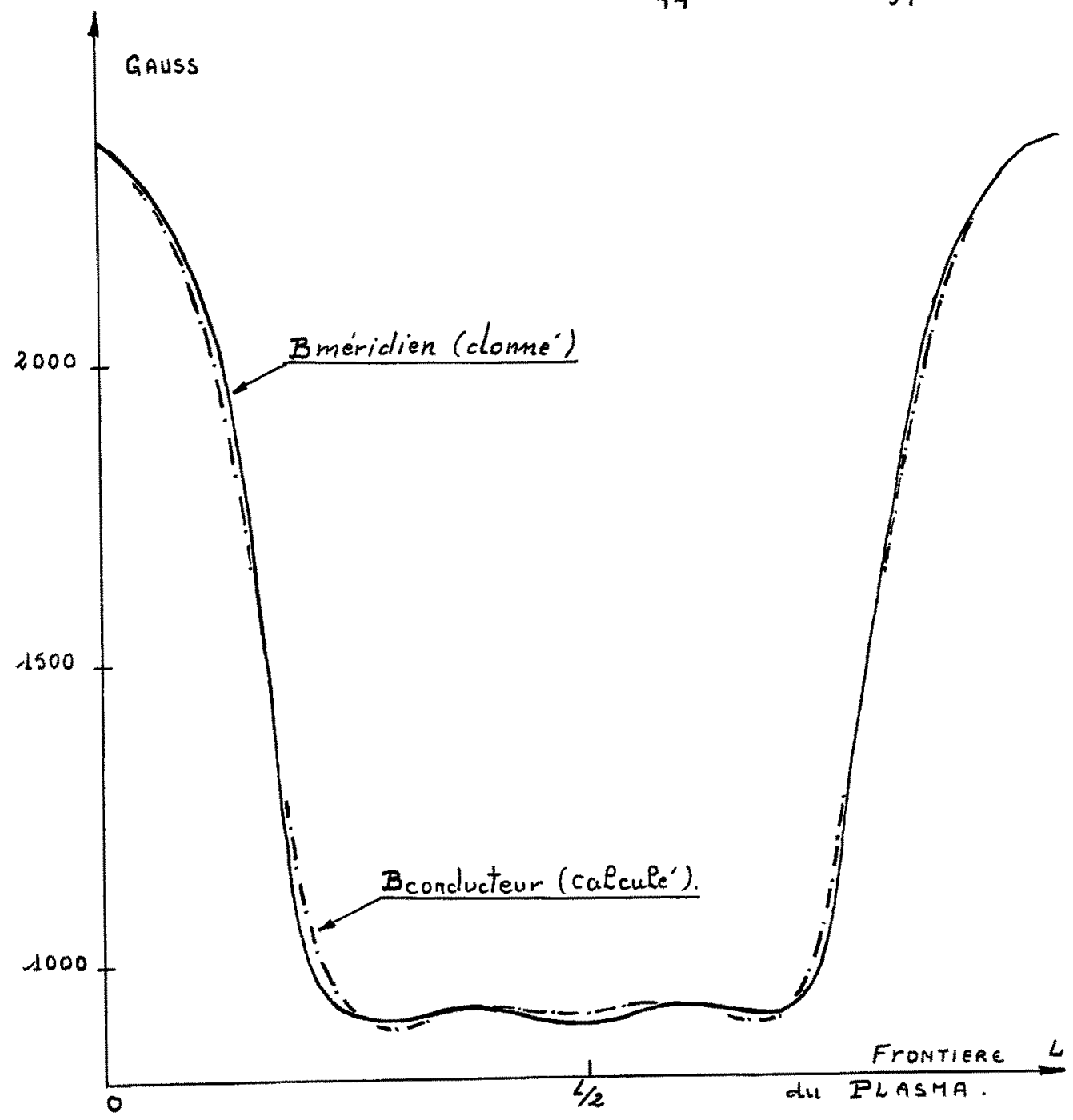




\section{BIBLIOGRAPHIE}

1.

[1] I.A. ARTSIMOVICH

TOKOMAK Devices

Nuclear Fusion, 12 (1972), p. 215-252

[2] G. LAVAL, H. LUC, E.K. MASCHKE, C. MERCIER, R. PELLAT

Equilibre stabilisé et diffusion d'un plasma torique à section transversale non circulaire.

Fourth Conference on plasma physics and controlled nuclear fusion research

(MADISON 1971), IAEA Vienne 1971, Vol. 1. 2 p. 507

[3] A.V.BORTNIKOV at al

The first experimental results in finger ring TOKOMAK

Sixth European Conference on controlled fusion and plasma Physics, MOSCOU 1973

11.

[1] J.L. LIONS, E. MAGENES

Problèmes aux limites non homogènes, DUNOD

[2] AGMON, DOUGLIS, NIRENBERG

Estimate near the boundary for solution of elliptic partial differential equations

Comm. pure applied Math. (12) 1959

[3] J.L. LIONS

Contrôle oprimai de systèmes gouvernés par des équations aux dérivées partielles, DUNOD

[4] Article à parâtre

avec C. MERCIER et SOUBBARAMAYER 